\title{
Detection of cancer antigens (CA-125) using gold nano particles on interdigitated electrode-based microfluidic biosensor
}

\author{
Bharath Babu Nunna', Debdyuti Mandal', Joo Un Lee², Harsimranjit Singh'1, Shiqiang Zhuang', \\ Durgamadhab Misra ${ }^{3}$, Md Nasir Uddin Bhuyian ${ }^{3}$ and Eon Soo Lee ${ }^{1^{*}}$ (1)
}

\begin{abstract}
Integrating microfluidics with biosensors is of great research interest with the increasing trend of lab-on-the chip and point-of-care devices. Though there have been numerous studies performed relating microfluidics to the biosensing mechanisms, the study of the sensitivity variation due to microfluidic flow is very much limited. In this paper, the sensitivity of interdigitated electrodes was evaluated at the static drop condition and the microfluidic flow condition. In addition, this study demonstrates the use of gold nanoparticles to enhance the sensor signal response and provides experimental results of the capacitance difference during cancer antigen-125 (CA-125) antigen-antibody conjugation at multiple concentrations of CA-125 antigens. The experimental results also provide evidence of disease-specific detection of CA-125 antigen at multiple concentrations with the increase in capacitive signal response proportional to the concentration of the CA-125 antigens. The capacitive signal response of antigen-antibody conjugation on interdigitate electrodes has been enhanced by approximately 2.8 times (from 260.80 to $736.33 \mathrm{pF}$ at $20 \mathrm{kHz}$ frequency) in static drop condition and approximately 2.5 times (from 205.85 to $518.48 \mathrm{pF}$ at $20 \mathrm{kHz}$ frequency) in microfluidic flow condition with gold nanoparticle-coating. The capacitive signal response is observed to decrease at microfluidic flow condition at both plain interdigitated electrodes (from 260.80 to $205.85 \mathrm{pF}$ at $20 \mathrm{kHz}$ frequency) and gold nano particle coated interdigitated electrodes (from 736.33 to $518.48 \mathrm{pF}$ at $20 \mathrm{kHz}$ frequency), due to the strong shear effect compared to static drop condition. However, the microfluidic channel in the biosensor has the potential to increase the signal to noise ratio due to plasma separation from the whole blood and lead to the increase concentration of the biomarkers in the blood volume for sensing.
\end{abstract}

Keywords: Biosensor, Interdigitated gold electrodes, Microfluidic channel, Gold nanoparticles, Capacitance measurements

\section{Introduction}

An electrical biosensor detects biomolecular reactions by measuring changes in electrical properties like voltage, current, impedance, capacitance etc., [1]. Measuring capacitance has advantages such as high sensitivity to small changes in dielectric parameters, the possibility

\footnotetext{
${ }^{*}$ Correspondence: eonsoo.lee@njit.edu

${ }^{1}$ Advanced Energy Systems and Microdevices Laboratory, Department of Mechanical and Industrial Engineering, New Jersey Institute of Technology, 200 Central Avenue, Rm MEC 327, Newark, NJ 07102-1982, USA

Full list of author information is available at the end of the article
}

of minimizing the sensor size, and low power consumption requirement [2]. The integration of microfluidics and electrical immuno biosensing has growing demand due to its potential to reduce processing time and have low reagent consumption [3]. Bange et al. [4] was the first group to integrate microfluidics into electrochemical protein immune assays. The immuno biosensing on the microfluidic platform helped to make the electrochemical biosensing assays portable which allows the sensing mechanism to be easily implemented in point of care devices [5]. The implementation of the biosensing in the microchannels significantly reduces the sample 
requirement from milliliter $(\mathrm{ml})$ to microliter $(\mu \mathrm{l})$. A low sample volume is highly desired for the bodily fluid samples such as blood. The incorporation of the microfluidic platform on the biosensor provide the feasibility of expanding the sensor to multiplex assay to detect the panel of protein biomarkers that minimize the false positive and false negative scenarios in cancer diagnosis which commonly arise from measuring a single biomarker [6-8]. Though there has been many researchers reported on the integration of the microfluidics to the immunosensors, most of the studies are performed on microfluidic flow driven by the external pumps and flow control devices $[9,10]$. In this current study the flow in the microchannel is self-driven and controlled by altering the hydrophilicity of the microchannel. The primary factors contributing to the microfluidic biosensors performance are probe immobilization, specific binding, and the fundamental limits of probe affinity. Due to the tight confinement of the flow of the antigen solution in the microscale, the flows in the microchannel exerts high shear stresses on the surface of the microchannel and influence the stability of the immobilized antibodies on the surface $[11,12]$. So the study of the sensing signal response during the flow condition attracted many researchers to develop novel techniques of antibody immobilization for enhanced stability.

For enhanced binding capabilities of the biomolecules on the sensing platform, nanoparticles and nanotechnology have attracted attention in recent years for their potential applications. The recent technology advancement in the microfluidic and nano technology present multiple opportunities for the development of lab-onchip (LOC) systems to perform a complete set of biomedical assays to achieve the low cost, highly sensitive point-of-care diagnostics [13-16]. Nanoparticles are favorable for biosensing, due to their potential for unique surface chemistry, electrical properties, and being in the same size range as biomolecules. Certain nanoparticles are biocompatible, which enables them to bond with various functional groups like proteins, ligands, peptides, DNA, fatty acids and plasmids for serving the sensing purpose $[17,18]$. There are various noble metals like gold, silver, palladium, rhodium, platinum etc. which are biocompatible [19]. Although many noble metals can function as biosensors, gold nanoparticles show promise for biosensing due to their unique surface chemistry, high electron densities, chemical inertness, and their possession of good electrical and optical properties $[4,6-8,20-48]$. The gold nanoparticles help in improving the sensitivity and actively targets the biomarker as it provides the platform for high surface to volume ratio $[21,22]$. In label free biosensors, the capacitive measurements are expected to change significantly with different properties such as the dielectric constants [1]. CA-125, a widely used biomarker for detection and monitoring of the ovarian cancer, is an exceptionally large protein (200 to $2000 \mathrm{kDa}$ due to high variable glycosylation) [23, 24]. As the capacitance measurement during the biological interactions is directly influenced by the physiochemical properties of an individual protein, the study of the CA-125 protein detection with its unique properties has gained importance in the research of biosensing.

Daniels et al. [25] has reported that the microfluidic biosensor performance can be enhanced with further research on the probe immobilization techniques. An improved understanding of the relationship between the antibody binding and the capacitance change would enable improved biosensor design and sensitivity. Though there are multiple recent studies performed by researchers, such as Goddard and Erickson [26], who have studied the stability of the biomolecules under the shear flow condition, most of them are limited to the study of DNA immobilization. Though the major targeted probes of the biosensors are proteins and DNA, there is very limited study reported on the antibody immobilization under the shear flow conditions during self-driven flow. This paper reports the sensitivity study of the antigen detection under shear flow condition during self-driven flow of antigen solution, when immobilized with the gold nano particles (GNPs). The sensitivity study performed in this paper on the capacitive signal response of the gold nano particle coated interdigitated electrodes compared to the plain interdigitated electrodes during the antigen-antibody conjugation provide the experimental evidence to the research of nano particle influence on the biosensor research. The study of sensing signal response of antigen-antibody conjugation with multiple concentrations of the antigens discussed in this paper, pave the way for study of sensitivity and specificity of the detection. Also, the change in the sensing signal due to the microfluidic flow of antigen solution when compared to static drop condition during the antigen-antibody conjugation aid the microfluidic biosensing researchers to understand the influence of the shear stress on the biosensing in microchannel.

\section{Methods/experimental}

\subsection{Chemicals and apparatus}

Thiourea $\left(\mathrm{CH}_{4} \mathrm{~N}_{2} \mathrm{~S}\right)$, phosphate buffer saline (PBS), 1-ethyl-3-(-3-dimethylaminopropyl) carbodiimide (EDC), $N$-hydroxysuccinimide (NHS), and carboxylic functionalized (Lipoic acid) gold nanospheres were purchased from NanoComposix (USA). The CA-125 monoclonal antibodies and the CA-125 antigens were bought from Meridian Life Science. The polydimethylsiloxane 
(PDMS) base and curing agent were bought from Fisher Scientific.

\subsection{Interdigitated electrodes fabrication}

The interdigitated electrodes were patterned using a photolithography process. A silicon wafer with an oxide layer was used as the substrate. A positive tone photoresist, PMMA- 6 was added on top of the silicon wafer and spin coated for $60 \mathrm{~s}$ at $3000 \mathrm{rpm}$. The photoresist coated silicon wafer was exposed with e-beam lithography using JEOL JBX6300-FS Electron Beam Lithography equipment from Brookhaven National Laboratory. After the e-beam exposure, the patterned silicon wafer was developed using 1:3 of MIBK: IPA for $60 \mathrm{~s}$ and further washed with IPA for another $60 \mathrm{~s}$ followed by drying with nitrogen gas. A layer of Titanium approximately $15 \mathrm{~nm}$ thick was deposited on top of the patterned silicon substrate. The purpose of this procedure is to enhance the adhesion of gold on the silicon wafer. Subsequently, a gold layer $95 \mathrm{~nm}$ thick was deposited on top of the Titanium coated silicon substrate using high vacuum e-beam metal evaporator (Kurt J. Lesker PVD-75 Evaporator). Followed by the metal depositions, a lift-off process was performed where the sacrificial positive photo resist was removed by cleaning the substrate in an acetone ultrasonic bath for $2 \mathrm{~min}$ and then followed by rinsing with IPA and de-ionized water to prevent redeposit ion. Figure 1 shows the AFM image of the plain interdigitated electrodes.

\subsection{Insulation of electrodes and addition of gold nanoparticles}

The interdigitated gold electrodes were washed multiple times with ethanol and de-ionized water and dried with nitrogen gas before addition of the self assembled

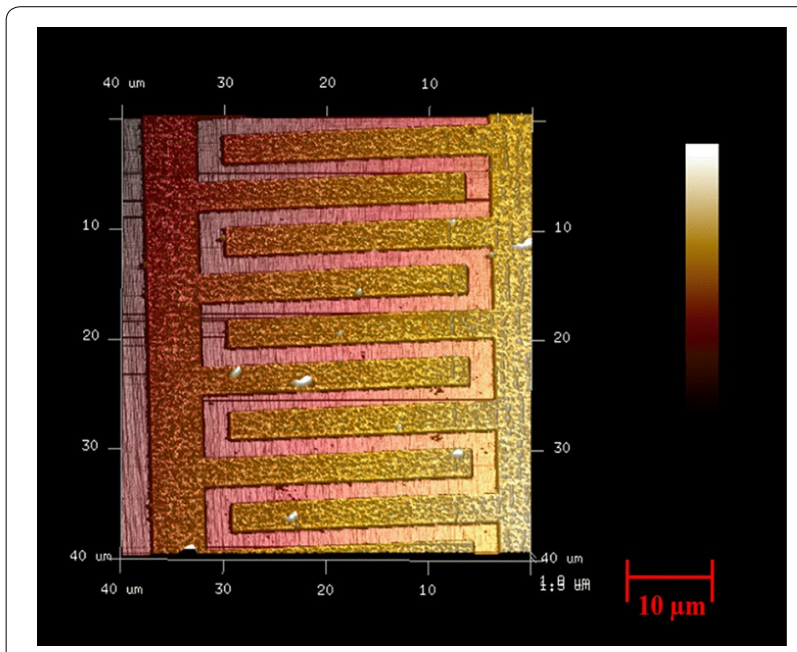

Fig. 1 AFM image of the plain electrodes monolayer (SAM) layer. The interdigitated gold electrodes surface was coated with SAM by submerging the sensor in $50 \mathrm{mM}$ thiourea solution overnight $(\sim 12 \mathrm{~h})$. The interdigitated electrodes were rinsed with ethanol and de-ionized water and dried with nitrogen gas. The formation of the SAM layer was confirmed by taking electrical measurements using a two-point electrical probe station. Furthermore, the AFM image of the electrodes confirmed the formation of the SAM layer. The carboxylic encapsulated gold nanoparticles having $5 \mathrm{~nm}$ size were incubated on the surface of the SAM modified sensor for $12 \mathrm{~h}$. After the incubation step, the gold nanoparticles surface was activated using $50 \mathrm{mM}$ of EDC and NHS. This process enables the antibodies to attach covalently to the carboxylic gold nanoparticles [27]. The surface activation of the carboxylic gold nanoparticles is shown in Fig. 2.

\subsection{Immobilization of CA-125 antibodies}

The sensor surface was washed using PBS solution and dried with nitrogen gas for removal of excess gold nanoparticles. Following this step, the CA-125 antibodies were immobilized on top of the surface activated gold nanoparticles by incubating it with $0.5 \mu \mathrm{l}$ of $7 \mathrm{mg} / \mathrm{ml} \mathrm{CA-125}$ antibodies in PBS solution, for $4 \mathrm{~h}$. The incubation step took place at $4{ }^{\circ} \mathrm{C}$. The sensor surface was rinsed using PBS solution and the non-reacted groups on the sensor surface were blocked by addition of approximately $1 \mu \mathrm{l}$ of ethanolamine on top of the modified sensor for $1 \mathrm{~h}$. The sensor was further cleaned with PBS and de-ionized water.

\subsection{Fabrication of PDMS microchannel}

The fabrication of the microchannel is primarily performed in two steps. In the first step, a Si-mold with the required microchannel pattern is fabricated. A positive photoresist (SPR 955) is used to have the photoresist remain only at the microchannel area after UV exposure using the UV mask aligner (since the mask is chromed at the microchannels). The Si-wafer is then etched with deep reactive ion etching (DRIE) to a depth of $107 \mu \mathrm{m}$ except at the microchannel surface area that is covered with photoresist. By etching the surface unprotected by the photoresist, the negative of the microchannels protrude from the surface of Si-wafer.

The microchannels are fabricated out of polydimethylsiloxane (PDMS) using the Si-wafer mold with negative microchannel. The PDMS base and curing agent are mixed in 10:1 ratio and degassed in the vacuum chamber. The PDMS mixture is poured on the Si-wafer with microchannel and baked for $45 \mathrm{~min}$ at $100^{\circ} \mathrm{C}$. The PDMS is then peeled from the $\mathrm{Si}$-wafer and treated with plasma for $100 \mathrm{~s}$ to convert the hydrophobic nature of PDMS 


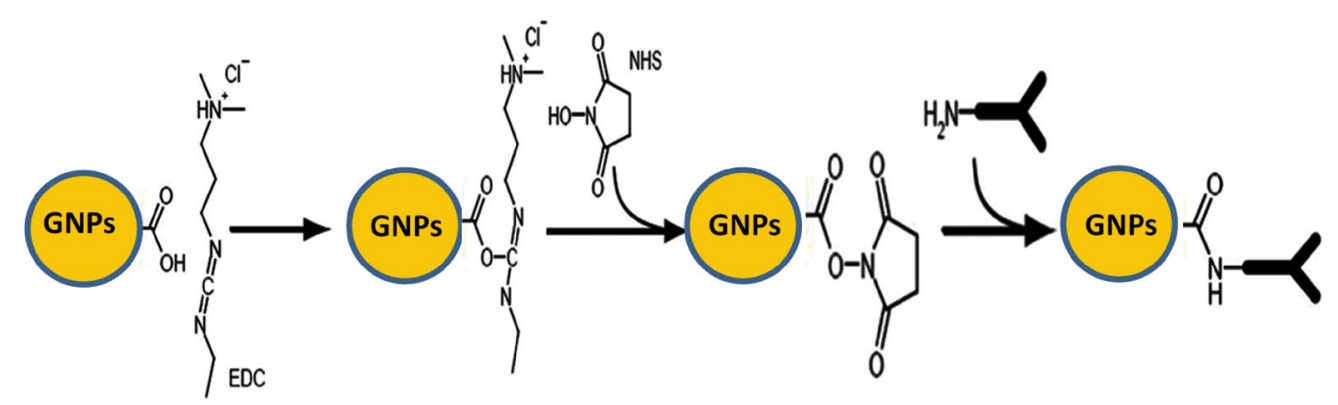

Fig. 2 Surface activation process of the carboxylic functionalized gold nanoparticles for antibodies binding

$\mathbf{a}$

(i)

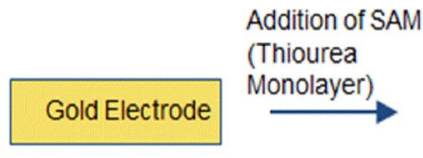

(v)

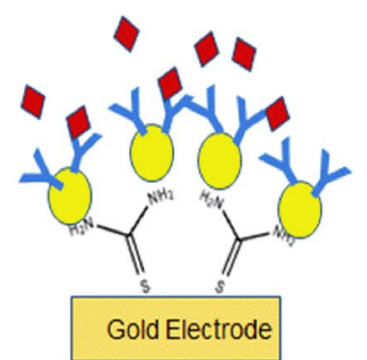

(ii)

(iii)

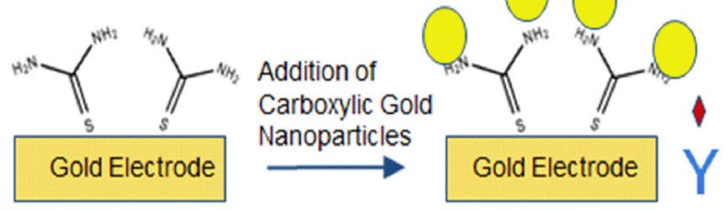

- CA-125 Antigens

CA-125 Antibodies

Carboxyl Group Gold Nanoparticles

Thiourea Monolayer

(iv)

Addition of CA125 Antigens
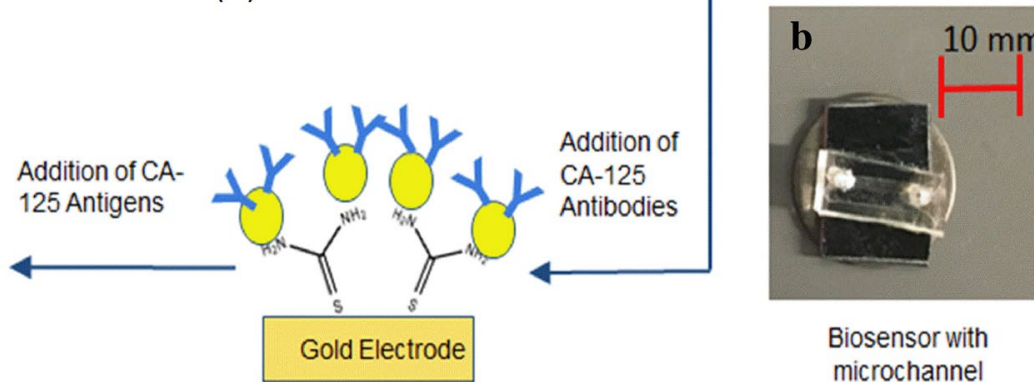

Fig. 3 a Schematic representation at various stages of biosensor fabrication: (i) Bare electrodes (ii) SAM layer on the bare electrodes (iii) immobilized gold nano particles on the SAM layer (iv) Antibody immobilization on the electrodes (v) antigen-antibody conjugation on the electrodes. b Real image of the biosensor with microchannel

to hydrophilic. The inlet and outlet ports of the microchannel are holes of $500 \mu \mathrm{m}$ diameter. The PDMS is then attached to the Si-wafer with the nano circuit to close the channel.

\subsection{Addition of CA-125 antigens}

The biosensor was exposed to CA-125 antigens in both the static and microfluidic flow conditions. The static condition was achieved by placing a drop directly on the sensor, whereas the flow condition was achieved by placing a drop on the flow inlet, and it followed the channel to encounter the sensor [45-47]. A $5 \mu \mathrm{l}$ droplet was used in both cases. Figure 3 shows the schematic of the different layers in the biosensor including the SAM layer fabrication, attachment the gold nano particles, immobilization of the CA-125 antibodies and CA-125 antigenantibody conjugation.

\subsection{Electrical measurements}

All the electrical measurements in this experiment were taken using a two-point probe station and the capacitance was measured using an Agilent 4284A Precision LCR meter. The selected frequency range was between 10 and $100 \mathrm{kHz}$ with $10 \mathrm{kHz}$ steps. The capacitive values were measured for (a) bare electrodes (b) after insulation of the electrodes by SAM layer, (c) after addition of carboxylic functionalized gold nanoparticles, (d) after the immobilization of the CA-125 antibodies, (e) after the interaction of the PBS solution with 
the sensor surface with the immobilized CA-125 antibodies, (f) after conjugation of CA-125 antigens and antibodies when antigens solution with different concentration of CA-125 antigens were dropped on the sensing surface, and (g) after conjugation of CA-125 antigens and antibodies when antigens solution flows through the microchannel. All the capacitive measurements were done at $100 \mathrm{mV}$ amplitude with the $0.5 \mathrm{~V}$ voltage during this experiment.

\section{Results and discussion}

\subsection{Interdigitated electrodes}

The interdigitated electrodes produce certain electric fields when voltage is applied, for capacitive measurements. The interdigitated electrodes provide greater effective surface area within the same volume or space which would drastically reduce the sensing setup and cost as compared to other capacitive measurement systems [28-31]. The electric field produced by the interdigitated electrodes is within the nanoscale range, which falls in the region of interest as the size of the antigens and antibodies lies in this range [32, 34]. The dielectric properties of the medium between the interdigitated electrodes provide the electrical information such as conductivity, permittivity, capacitance and impedance. The electric field lines produced by the interdigitated electrodes depend on the electrical input, dielectric medium, and the geometry of the electrodes.

The effective surface area of the electrode that contributes to the capacitance is, 'Top' surface area and 'Side' surface area as shown in Fig. 4a. The net capacitance ' $\mathrm{C}$ ' of the active surface area (Top + Side) of the electrodes in the comprised capacitance model can be determined by Eq. (1),

$$
\mathrm{C}=\varepsilon_{\mathrm{r}_{\mathrm{T}}} \cdot \varepsilon \boldsymbol{\varepsilon} \cdot\left(\frac{\mathrm{A}_{\mathrm{eff}_{\mathrm{Top}}}}{\mathrm{d}_{\mathrm{eff}_{\mathrm{Top}}}}\right)+\varepsilon_{\mathrm{rS}_{\mathrm{S}}} \cdot \varepsilon \boldsymbol{\delta} \cdot\left(\frac{\mathrm{A}_{\mathrm{eff}}}{\mathrm{d}_{\text {Side }}}\right)
$$

where $\varepsilon_{\mathrm{r}_{\mathrm{T}}}$ and $\varepsilon_{\mathrm{rS}}$ is the relative permittivity of the material on the top and side surfaces of the electrode, $\varepsilon 0$ is the

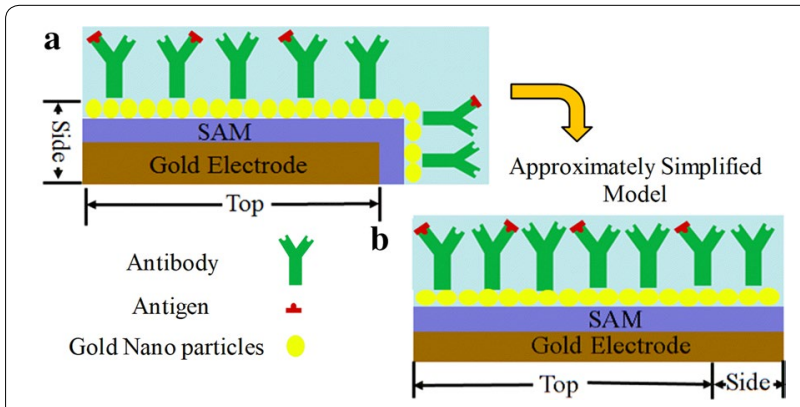

Fig. 4 a Schematic of the electrode with active 'Top' and 'Side' surfaces of the and $\mathbf{b}$ the approximately simplified model of $\mathbf{a}$ with single surface model for both Top and Side in this analysis vacuum permittivity, $\mathrm{A}_{\text {eff }}$ Top and $\mathrm{A}_{\text {eff }}$ Side are the effective surface areas on the top and side surfaces of the electrode, and $\mathrm{d}_{\mathrm{eff}}$ Top is the effective distances neighboring two top surfaces and $\mathrm{d}_{\text {eff }_{\text {Side }}}$ is between the adjacent two side surfaces of the electrode.

When the SAM, Antibody and Antigen/Antibody layers are assumed to be homogenous over the surface of electrode, the capacitance of the circuit can be calculated from the equivalent model, with the single surface (Top + Side) as shown in Fig. 4b.

Hence, the net capacitance ' $\mathrm{C}$ ' of the approximately simplified model can be calculated as per Eq. (2),

$$
\mathrm{C}=\varepsilon_{\mathrm{r}} \cdot \varepsilon \boldsymbol{o} \cdot\left(\frac{\mathrm{A}_{\mathrm{eff}}}{\mathrm{d}_{\mathrm{eff}}}\right)
$$

And,

$$
\mathrm{C}=\mathrm{C}_{\text {Top }}+\mathrm{C}_{\text {Side }}
$$

where,

$$
\begin{aligned}
& \mathrm{C}_{\text {Top }}=\varepsilon_{\mathrm{r}_{\mathrm{T}}} \cdot \varepsilon \boldsymbol{o} \cdot\left(\frac{\mathrm{A}_{\text {Top }}}{\mathrm{d}_{\mathrm{Top}}}\right) \\
& \mathrm{C}_{\text {Side }}=\varepsilon_{\mathrm{r}_{\mathrm{S}}} \cdot \varepsilon \boldsymbol{O} \cdot\left(\frac{\mathrm{A}_{\text {Side }}}{\mathrm{d}_{\text {Side }}}\right)
\end{aligned}
$$

By assuming,

$$
\begin{aligned}
& \varepsilon_{\mathrm{r}} \approx \varepsilon_{\mathrm{r}_{\mathrm{T}}} \approx \varepsilon_{\mathrm{rs}} \\
& \mathrm{A}_{\mathrm{eff}} \approx \mathrm{A}_{\mathrm{eff}}{ }_{\text {Side }}+\mathrm{A}_{\mathrm{eff}_{\mathrm{Top}}}
\end{aligned}
$$

With Eq. (2) to Eq. (7) $d_{\text {eff }}$ can be written as,

$$
\mathrm{d}_{\mathrm{eff}} \approx \frac{\mathrm{A}_{\mathrm{eff}_{\text {Side }}}+\mathrm{A}_{\mathrm{eff}_{\text {Top }}}}{\left(\frac{\mathrm{A}_{\mathrm{eff}}}{\mathrm{d}_{\text {eff }}}\right)_{\text {Side }}+\left(\frac{\mathrm{A}_{\mathrm{eff}}}{\mathrm{d}_{\mathrm{eff}}}\right)_{\text {Top }}}
$$

where, $\varepsilon_{\mathrm{r}}$ (as per Eq. (3)) is the relative permittivity of the material between the electrodes, $\varepsilon o$ is the vacuum permittivity, $A_{\text {eff }}$ is the effective overall surface area of top and side, and $d_{\text {eff }}$ is the effective overall distance between electrodes as shown in Fig. 5 as per Eq. (8).

\subsection{Surface characterization of different layers of the biosensor}

In this study, the purpose of the SAM layer is to insulate the electrodes and prevent them from short circuiting [33]. The functionality and the presence of the SAM layer are confirmed by AFM image of the Thiourea coated gold electrodes. Figure 6 shows the AFM image of the electrodes having Thiourea layer deposited on top of it. The increment in the net vertical height $(\sim 20 \mathrm{~nm})$ of the electrodes along with the surface 

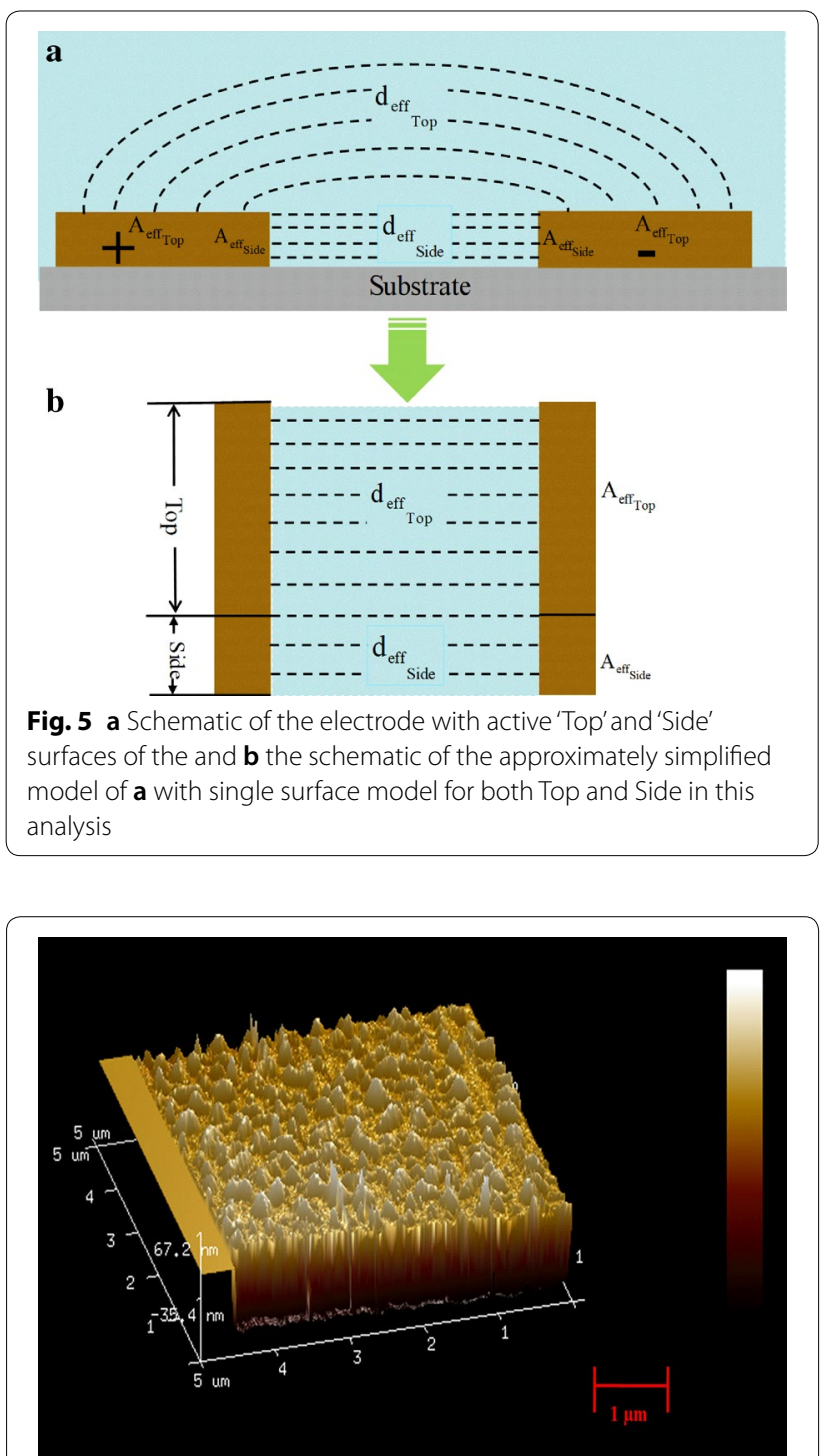

Fig. 6 The AFM image of the interdigitated gold electrode coated having SAM layer deposited on top

roughness confirms the formation of the SAM layer. The SAM layer insulation was also confirmed using electrical measurements. The carboxylic functionalized gold nanoparticles were incubated, and surface activated using EDC/NHS coupling. The CA-125 antibodies are then added and incubated on top of the gold nanoparticles. Figure 7 shows the AFM images of the gold nanoparticles and the antibodies present on top of the gold nanoparticles.

\subsection{CA-125 antigen solution flow in microchannel} In this experiment, a microchannel with the width of ' $W$ ' $(300 \mu \mathrm{m})$ and depth of ' $\mathrm{h}$ ' $(107 \mu \mathrm{m})$ is used to generate the microfluidic flow on the sensing platform as shown in Fig. 8a. The microchannel is fabricated using PDMS, which is hydrophobic in nature. The hydrophobic nature of PDMS is converted to hydrophilic using the plasma treatment. The flow of the antigen solution in the microchannel due to the capillary effect generates a shear on the sensing surface. The shear stress at the surface of sensing is defined by the change in the antigen solution flow velocity $\left(U_{x}\right)$ with respect to the channel height at the channel surface $(y=0)$, by assuming that the flow of the antigen solution as the poiseuille flow in the infinite parallel plates due to the high aspect ratio and the insignificant side wall effect.

$$
\tau=\left.\mu \frac{\partial \mathrm{Ux}}{\partial \mathrm{y}}\right|_{\mathrm{y}=0} \approx \mu \frac{6 \mathrm{Q}}{w h^{2}}
$$

where the flow rate of the antigen solution is measured as $\mathrm{Q}(0.2 \mu \mathrm{l} / \mathrm{s})$ using a high-speed camera (Phantom V-7.3), with the dynamic viscosity $(\mu)$ of the antigen solution $\left(8.8 \times 10^{-4}\right.$ pa s) the width of the microchannel is $\mathrm{W}$ $(300 \mu \mathrm{m})$, the depth of the microchannel is $\mathrm{h}(107 \mathrm{um})$ as shown in Fig. 8b. Thus, the shear stress $(\tau)$ is calculated as 0.307 pa as per Eq. (9). The shear stress has influences on the stability of the immobilized CA-125 antibodies on top of the sensing surface with gold nano particles and the corresponding effect on sensitivity are explained in detail in Sect. 3.4.5 of the paper.

\subsection{Electrical characterization \\ 3.4.1 Capacitance measurement of different layers of biosensor}

The capacitance is measured at various stages consisting of different sub-layers. All the measurements were taken using a two-point probe station and the dielectric parameters were calculated using Agilent 4284A Precision LCR meter. The frequency range was taken from 10 to $100 \mathrm{kHz}$ for all the sub-layers using $10 \mathrm{kHz}$ steps.

Figure 9 shows the plot of capacitance variation over frequency for different layers of the sensor. The highest capacitance of the bare interdigitated electrodes was $9.38 \mathrm{pF}$ at $10 \mathrm{kHz}$ and the lowest was $8.70 \mathrm{pF}$ at $100 \mathrm{kHz}$. The capacitance values of the SAM layer (Thiourea) was observed to be lower than the bare electrodes. The highest value recorded was $9.06 \mathrm{pF}$ at $10 \mathrm{kHz}$ and the lowest was $8.47 \mathrm{pF}$ at $100 \mathrm{kHz}$. The potential reason is the higher charge transfer resistance of the SAM layer which directly affects the real part of the impedance. This increase in the resistance directly influences and increases the net impedance. Because of this phenomenon, the net capacitance of the circuit decreases over frequency [35, 36]. The higher permittivity of the gold nanoparticles resulted in higher capacitance than that of both bare electrodes and the SAM 

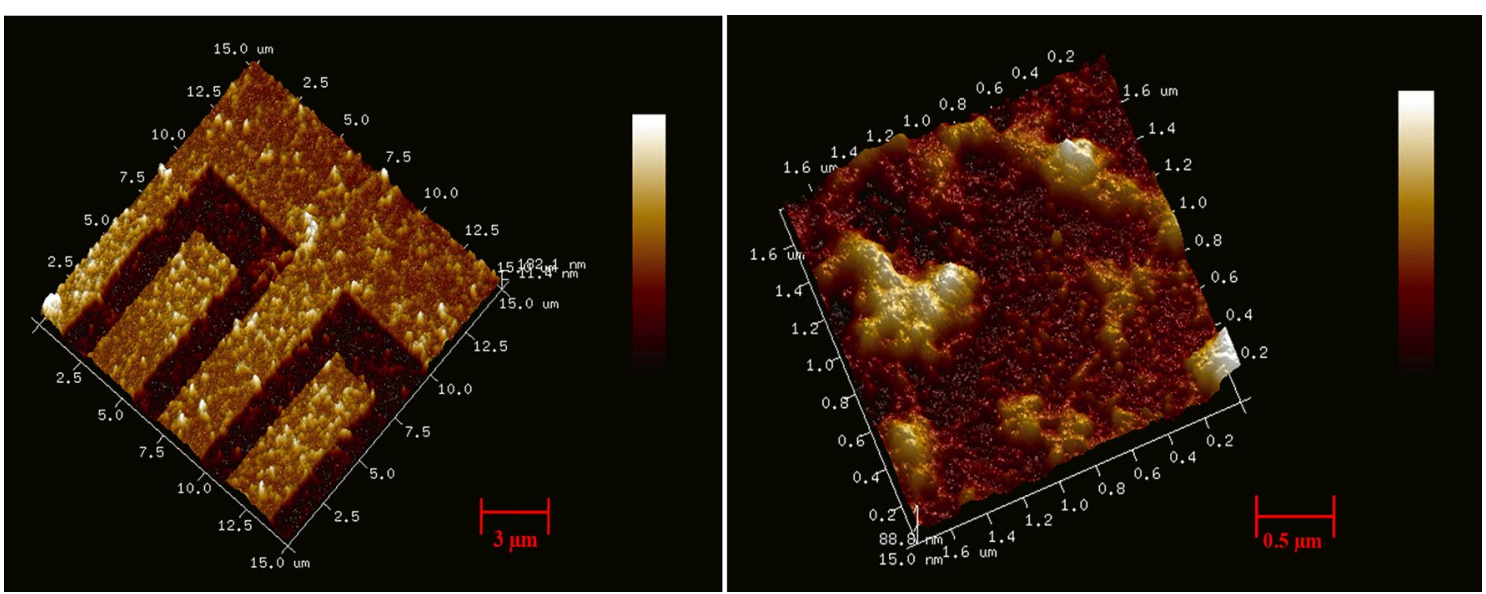

Fig. 7 AFM images of the Gold nanoparticles present on top of gold electrodes (Left) and the CA-125 antibodies present on top of the gold nanoparticles in the sensor platform (Right)

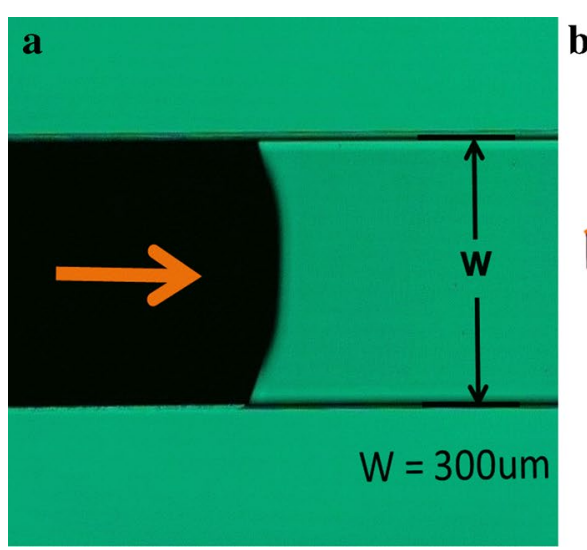

b $\mathrm{U}_{\mathrm{x}}=$ Change in flow velocity of antigen solution $\Upsilon=$ Shear rate at the sensing surface

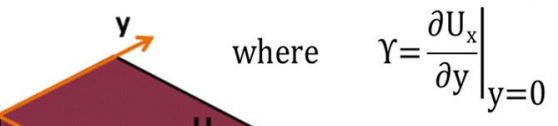

Fig. 8 a The image microchannel during the flow of antigen solution. b Schematic of the microchannel with the shear rate measurement

layer, giving values of $9.62 \mathrm{pF}$ at $10 \mathrm{kHz}$ and $8.76 \mathrm{pF}$ at $100 \mathrm{kHz}$. The capacitance measurement at the immobilized CA-125 antibody layer was $11.94 \mathrm{pF}$ at $10 \mathrm{kHz}$ frequency and then reduced by increasing frequency, down to $9.36 \mathrm{pF}$ at $100 \mathrm{kHz}$ frequency as shown in Fig. 9.

\subsubsection{Capacitance measurement of a CA-125 antigen conjugation with CA-125 antibodies immobilized on biosensor}

The study used a solution of PBS solvent with and without CA-125 antigens respectively. First for the baseline study, plain PBS solution without CA-125 antigens was measured with CA-125 antibodies coated on the nano electrodes. A drop of PBS solution (approximately $1 \mu \mathrm{l}$ ) was placed on the biosensor. The PBS solution capacitance measurements were taken in the frequency range from 10 to $100 \mathrm{kHz}$. The capacitance values almost

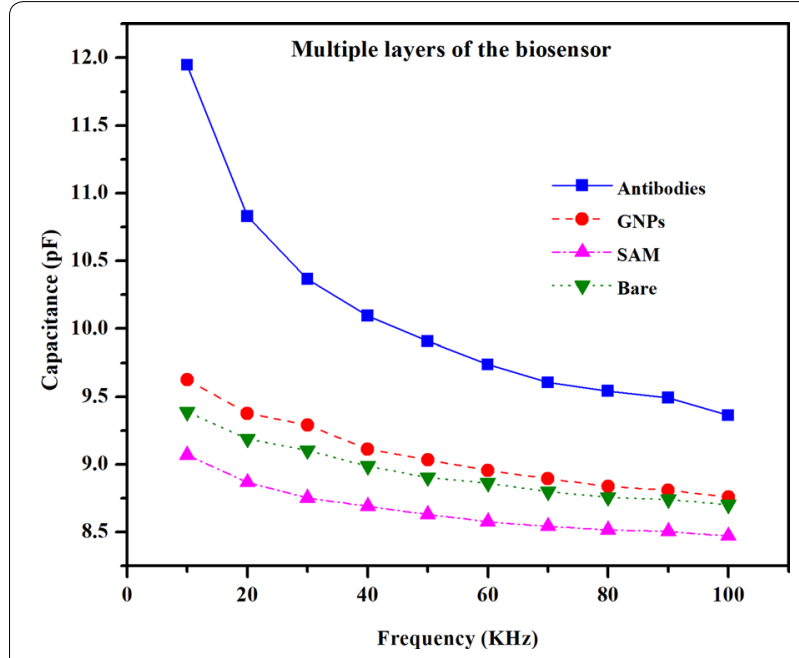

Fig. 9 Capacitance variation over frequency for different layers of the sensor 
remained unchanged over the entire frequency range. The capacitance curve of the plain PBS solution was regarded as the 'Baseline.' The highest and lowest capacitive values of the 'Baseline' curve were measured to be $96.90 \mathrm{pF}$ and $69.19 \mathrm{pF}$ respectively.

The next for CA-125 antigens case, a $1 \mu \mathrm{l}$ droplet of CA-125 antigen solution is placed on the biosensor. The capacitance measurements of the antigen and antibody conjugation are taken from the same range of frequency. The capacitive values of the CA-125 antigen solution case with CA-125 antigen-antibody conjugation, 'after AgAb conjugation' curve was measured to be $822.93 \mathrm{pF}$ at $10 \mathrm{kHz}$ and changed to $342.18 \mathrm{pF}$ at $100 \mathrm{kHz}$. The antigens and the antibodies interaction are very selective and specific. The specific antigen and antibody interactions form a complex which increases the net molecular size. The change in the size of the complex which has a unique property of electrical charge, disturb or interfere the distribution of the charges present in the dielectric medium. The antigen-antibody complex which has unique property of electrical charge creates a change in the distribution of charges within the dielectric region and forms a dipole moment. Because of this phenomenon, the polarization is created due to the dipole-dipole interaction within the dielectric interface [33, 34] substantially leads to a high increase in capacitance. Also, the dielectric values of each antigen-antibody complex over the range of frequencies have its unique variation. The measured impedance or capacitance of the biosensor varies with the change in dielectric properties on sensor surface. The change in the dielectric properties directly influences the change in the capacitance over a range of frequencies. The highest capacitance values were observed at $10 \mathrm{kHz}$ for both 'Baseline' and after the $\mathrm{Ag}-\mathrm{Ab}$ conjugation in the selected frequency range. The capacitance of the 'Baseline' is around $96.90 \mathrm{pF}$ and is increased to a value around $822.93 \mathrm{pF}$ after the antigens conjugation at $10 \mathrm{kHz}$ as shown in plot of Fig. 10. The significant change in the capacitance values represents the conjugation of the CA-125 antigens and antibodies.

\subsubsection{Capacitance measurement comparison of antigen- antibody interactions on plain interdigitated electrodes and gold nanoparticle layered interdigitated electrodes, respectively}

The gold nanoparticles-based sensing platform which was coated with gold nanoparticles on the interdigitated electrodes showed the enhanced capacitance during antigen-antibody interaction, compared to plain interdigitated electrodes, as seen in Fig. 11. Although there are various noble metals which can serve the purpose for biosensing, the gold nanoparticles are found to be promising and better for biosensing due to its unique surface

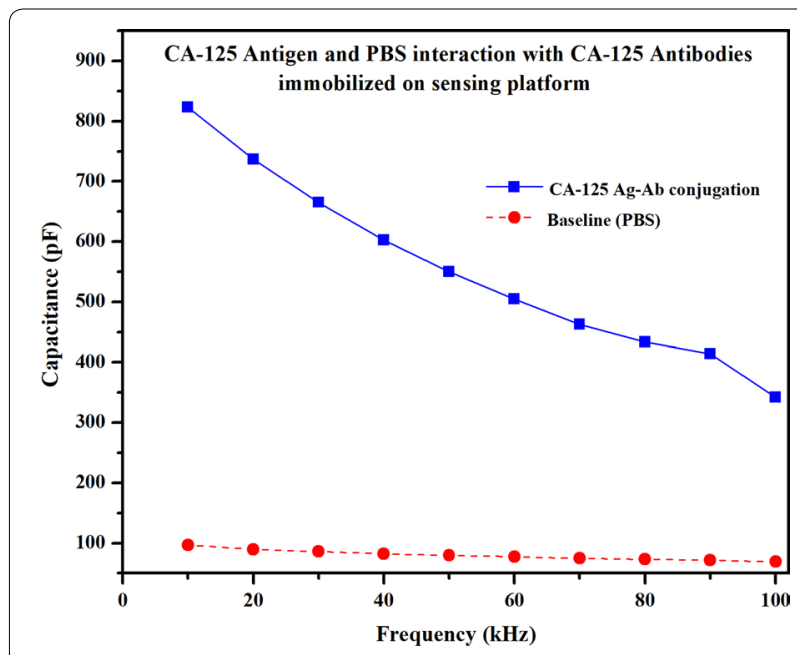

Fig. 10 Capacitance variation over frequency for both the cases: the baseline and CA-125 antigens during Ag-Ab conjugation

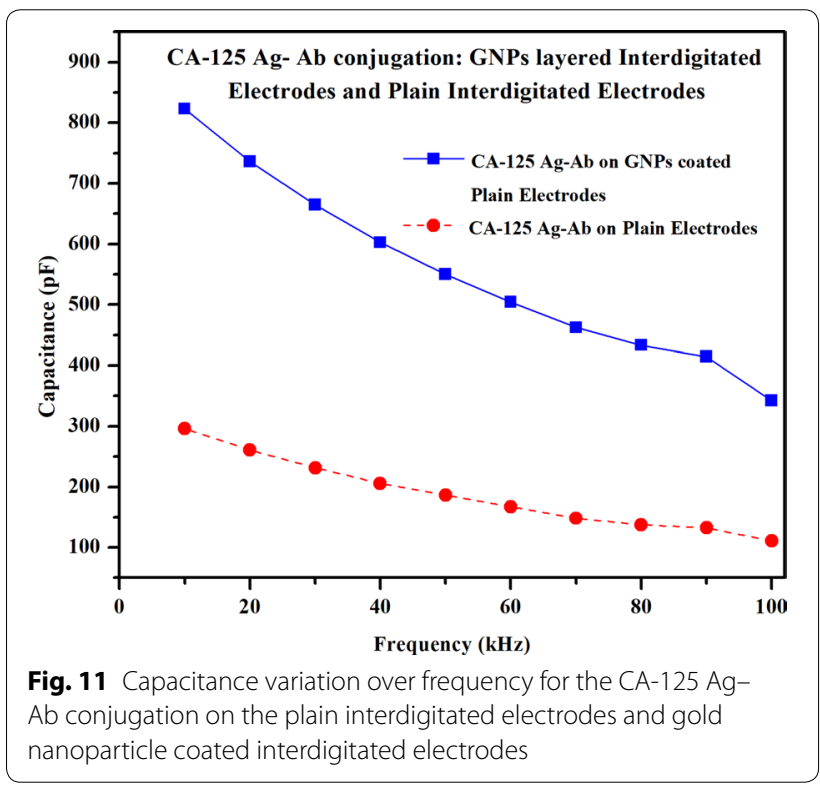

chemistry, high electron densities, chemical inertness and it possesses both good electrical and optical properties [20]. The carboxylic coated gold nanoparticles provide higher surface to volume ratio for the immobilization of the antibodies. The gold nanoparticles provide enhanced stability due to better orientational freedom for the antibodies attachment. This phenomenon not only provides better stability but also results in accumulating more antibodies for the antigen-antibody conjugation. The highest capacitance of both sensing platforms is measured at $10 \mathrm{kHz}$ and are found to be $822.93 \mathrm{pF}$ for gold nanoparticles coated interdigitated electrodes and 296.09 $\mathrm{pF}$ for plain interdigitated electrodes. The capacitance of 
the carboxylic gold nanoparticles is found to be almost three times the capacitance of the plain interdigitated electrodes. Two factors may be contributing to this phenomenon. The first explanation is due to the enhanced orientation freedom and higher surface to volume ratio of the gold nanoparticles as compared to the plain interdigitated electrodes. This characteristic results in increasing the net amount of the antibodies. As a result, the capacitance signal response is higher for gold nanoparticle coated interdigitated electrodes when compared to plain interdigitated electrodes [42-44]. Another explanation is the surface coverage of the carboxylic functionalized group on top of the nanostructure or nano-elements for covalent conjugation. Although the plain interdigitated electrode sensing mechanism also used covalent bonding for antibody binding, the gold nanoparticles provide much more surface area of the electrodes. This resulted in capturing a significantly greater number of antibodies using covalent bonding for the gold nanoparticles-based interdigitated electrodes.

\subsubsection{Capacitance measurement comparison of antigen- antibody interaction with multiple concentrations of antigens}

The capacitance signal response was captured during CA-125 antigen-antibody conjugation with different concentrations of CA-125 antigens, as shown in Fig. 12. The CA-125 antigens are aliquotted into different concentrations: $84,000 \mathrm{U} / \mathrm{ml}, 35,000 \mathrm{U} / \mathrm{ml}, 3500 \mathrm{U} / \mathrm{ml}$, and plain PBS (without CA-125 antigens). The CA-125 antigens of different concentrations were measured using the static

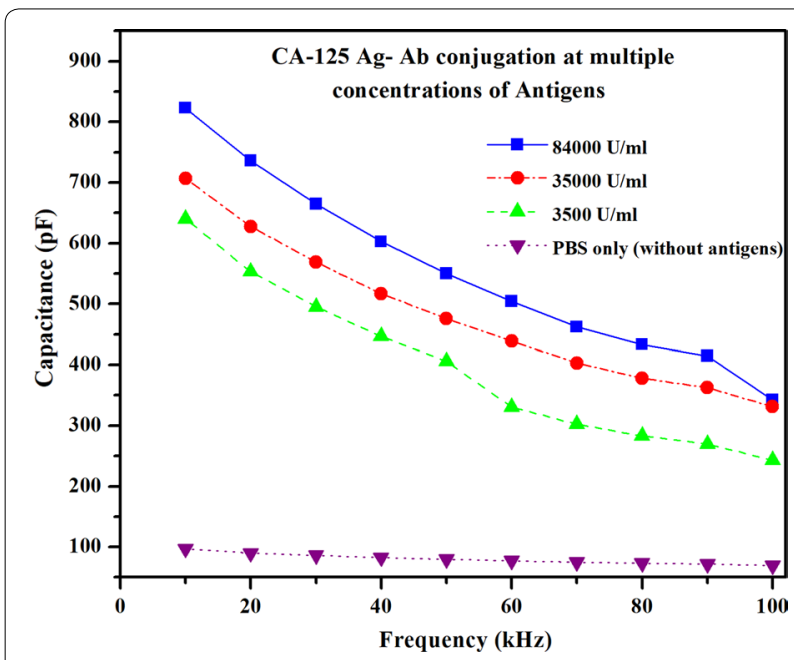

Fig. 12 Capacitance variation over frequency for the CA-125 Ag-Ab conjugation with various concentrations of CA-125 antigens and PBS without CA-125 antigens drop condition on the gold nanoparticle coated interdigitated electrodes. The antibodies immobilized on the gold nanoparticles interact with the CA-125 antigens and generate the corresponding capacitance signal response. The capacitance signal response during the antigen-antibody interaction is as high as $822.93 \mathrm{pF}$ with an antigen solution of $84,000 \mathrm{U} / \mathrm{ml}$ and the signal response drops to $706.91 \mathrm{pF}, 640.5 \mathrm{pF}$ and $96.90 \mathrm{pF}$ for the concentrations of $35,000 \mathrm{U} / \mathrm{ml}, 3500 \mathrm{U} / \mathrm{ml}$ and PBS (without antigens) respectively at $10 \mathrm{kHz}$ frequency. The capacitance signal response of the PBS solution without any antigens was captured to confirm that the change in signal difference is caused only due to the antigen-antibody interaction. The change in the capacitance signal is directly proportional to the concentration of the antigens in the sample. The signal response of antigen-antibody interaction for lower concentrations of antigens was observed due to the low number of antigens interacting with the antibodies that were immobilized on the gold nanoparticle based interdigitated electrodes. The capacitance signal for all the concentrations decreased as the frequency increased. As predicted by electrochemical theory, the change in the capacitance signal between two different concentrations of antigens at a frequency was almost same (over the frequency range of $10 \mathrm{kHz}$ to $100 \mathrm{kHz}$ ).

\subsubsection{Capacitance measurement comparison of CA-125 antigen-antibody conjugation at static and microfluidic flow condition}

The plot in Fig. 12 shows the variation in the signal response between the static condition and the microfluidic flow condition during the $\mathrm{CA}-125 \mathrm{Ab}-\mathrm{Ag}$ conjugation. The carboxylic gold nanoparticles sensing platform without the microchannel (static drop condition) resulted in consistently higher capacitance values because there is no external disturbance on antigen and antibody interaction whereas the microchannel flow has the external effect by the shear of the flow. The highest capacitance is recorded to be $822.93 \mathrm{pF}$ at $10 \mathrm{kHz}$ and the lowest is $342.18 \mathrm{pF}$ at $100 \mathrm{kHz}$.

\subsubsection{Microfluidic flow condition of the biofluid sample} with CA-125 antigens To understand the sensitivity variation due to the microfluidic flow, a biofluid sample with the exact same composition to the static drop condition was passed through the microchannel at a constant flow rate $(0.2 \mu \mathrm{l} / \mathrm{s})$ over the sensing platform. The capacitance values were measured during the antigen-antibody interaction when the biofluid was flowing in the microchannel. The capacitance measurement in the microfluidic flow condition during CA-125 antigen-antibody interaction was measured as $807.30 \mathrm{pF}$ at $10 \mathrm{kHz}$ frequency and gradually decreased to $234.51 \mathrm{pF}$ at $100 \mathrm{kHz}$ frequency as 


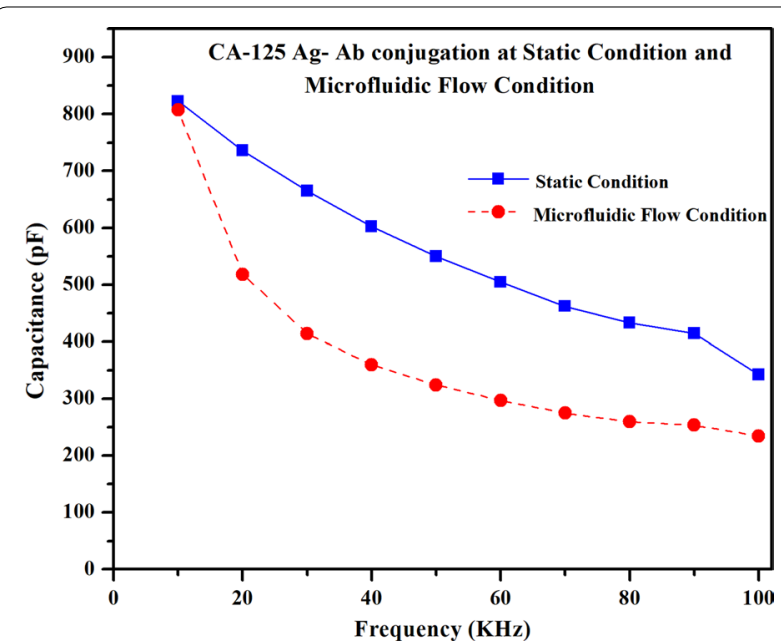

Fig. 13 Capacitance variation over frequency for gold nanoparticles under static and microfluidic flow condition

shown in Fig. 13. The capacitance measurement during the CA-125 antigen-antibody interaction has decreased from $822.93 \mathrm{pF}$ for the static drop condition to $342.18 \mathrm{pF}$ at $10 \mathrm{kHz}$ for the microfluidic flow condition as shown in Fig. 13.

The capacitance was recorded from the highest value of $807.30 \mathrm{pF}$ to the lowest value of $234.51 \mathrm{pF}$ within the frequency range from 10 to $100 \mathrm{kHz}$ during the flow of the antigen solution in the microchannel. The tight confinement of the microfluidic flow exerts high surface shear stress which impact the stabilization of the antibodies that are bonded to the sensing platform [37, 38]. The shear forces applied by the fluid on the antibodies that are bonded to the electrode sensing platform in the microchannel induce mechanical breakage of the weak bonds of the antibodies with the electrode [39-41]. The breakage of bonds of the antibodies with the sensing surface could influence the stability of the immobilization of antibodies. So due to the existence of shear in the microfluidic flow condition, the stability of the CA-125 antibody would be significantly lower, which could directly influence the sensitivity. So due to lack of any shear in 'static' condition, the stability of the CA-125 antibody was significantly higher and directly enhanced the sensitivity.

\subsubsection{Capacitance variation during CA-125 antigen} antibody interaction at different conditions (at $20 \mathrm{kHz}$ frequency) Figure 14 shows the change in the capacitance of antigen-antibody interaction at different conditions of the sensing platform (plain interdigitated electrodes and gold layered interdigitated electrodes) and different flow

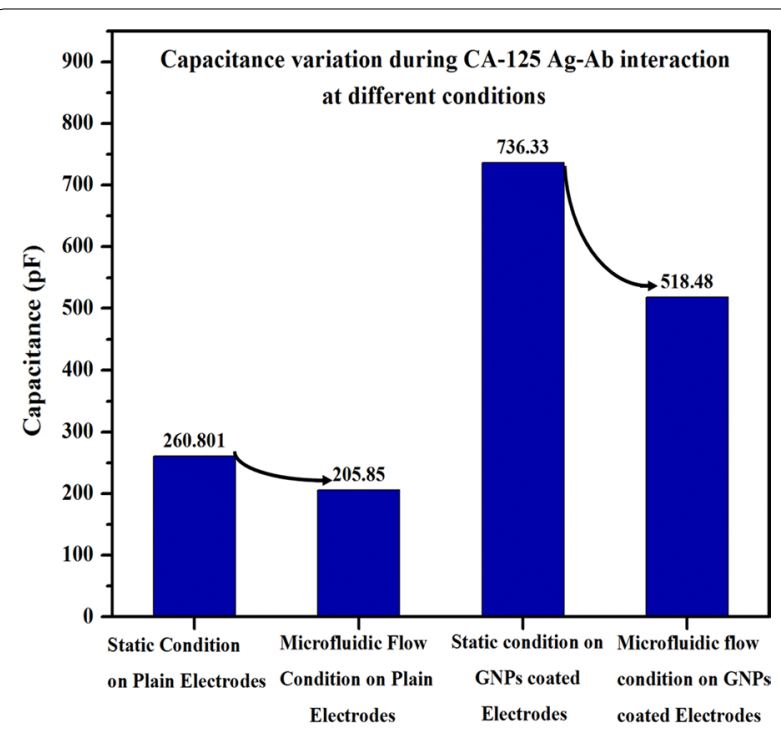

Fig. 14 Capacitance variation during $C A-125 \mathrm{Ag}-\mathrm{Ab}$ interaction at different conditions at $20 \mathrm{kHz}$ frequency

conditions (static drop condition and microfluidic flow condition) at $20 \mathrm{kHz}$ frequency.

As shown in Fig. 14, the change in the capacitance from the static drop condition to microfluidic condition for plain electrode is $54.95 \mathrm{pF}$ and for gold nanoparticle coated electrodes is $217.85 \mathrm{pF}$. As explained in the earlier sections, the gold nano particles (GNPs) coated electrodes has the higher sensing signal than the plain electrodes due to the enhanced antibodies immobilization on the gold nano particles with the high surface to volume ratio and orientation freedom. Also, GNPs has the higher resistance to shear flow than plain interdigitated electrodes for microchannel flow.

\section{Conclusions}

The incorporation of microfluidics with biosensing has the following advantages: multiplex assay and simultaneous separation of the targeted biomolecules for detection during the flow, for enhanced signal to noise ratio. However, it has the limitation due to the shear effect caused by the microfluidic flow on the antibodies immobilized on the sensing platform. In the current study, sensitivity variation due to microfluidic flow was established by detecting CA-125 antigens in biofluid using gold interdigitated electrodes. At static drop condition, the signal response of antigen-antibody conjugation in gold nanoparticle-coated interdigitated electrodes is approximately 2.8 times than in plain interdigitated electrodes. At $20 \mathrm{kHz}$ frequency, 
the signal response of plain interdigitated electrodes during antigen-antibody conjugation has increased from 260.80 to $736.33 \mathrm{pF}$ when the gold nano particles are coated on the plain interdigitated electrodes. At microfluidic flow condition, the signal response of antigen-antibody conjugation in gold nanoparticlecoated interdigitated electrodes is 2.5 times than in plain interdigitated electrodes. At $20 \mathrm{kHz}$ frequency, the signal response of plain interdigitated electrodes during antigen-antibody conjugation has increased from 205.85 to $518.48 \mathrm{pF}$ when the gold nano particles are coated on the plain interdigitated electrodes. Based on the measured results, the following conclusions can be made. (1) The functionality of the individual layers in the sensing platform is validated with the measured change in capacitance. (2) The gold nanoparticle coated interdigitated electrode has higher sensitivity than the plain interdigitated electrode during the CA-125 antigen antibody interaction. (3) The capacitive sensing signal response increased proportionally with the increase in concentration of the antigens during the antigen-antibody conjugation. (4) The effect of shear on the sensing signal response is evident given the lower capacitive signal during antigen-antibody conjugation in the microfluidic flow condition as compared to the static drop condition. The observed effect of shear stress in the microfluidic flow condition during the antigen-antibody conjugation can be mitigated by incorporating the following design changes in the sensing platform and microchannel. (i) The sensing platform with nano well-structure immobilized the antibody into each well, can reduce the shear effect during the microfluidic flow. (ii) The surface treatment to the microchannel for controlling the hydrophilicity of channel reduce the shear caused by the microfluidic flow significantly and thus the effect of the shear on sensing platform can be controlled. Though our focus was on isothermal microfluidic devices, the future work on evaluating the influence of thermal conditions on the sensing signal response in the microfluidic platform would provide additional information regarding the stability of the bioconjugation chemistries in thermocycling microfluidic biosensing applications.

\footnotetext{
Abbreviations

CA 125: cancer antigens 125; ml: milliliter; $\mu$ : microliter; DNA: deoxyribonucleic acid; kDa: kilodalton; GNPs: gold nano particles; PBS: phosphate buffer saline; PDMS: polydimethylsiloxane; PMMA: poly(methyl methacrylate); MIBK: methyl isobutyl ketone; IPA: isopropyl alcohol; SAM: self-assembled monolayer; EDC: 1-ethyl-3-(3-dimethylaminopropyl)-carbodiimide; NHS: N-hydroxysulfoxuccinimide; mg: microgram; DRIE: deep reactive ion etching; AFM: atomic force microscope.
}

\section{Authors' contributions}

BBN and DeM have contributed to sample preparation, data curation, data analysis, and original draft writing. BBN and ESL have contributed for review and editing the manuscript. JUL has contributed to procuring experimental resources, data acquisition and analysis. HS and SZ have contributed to procuring the resources and samples, and reviewing and editing the manuscript according to journal specifications. DuM, MNUB and BBN have contributed to the software support for acquiring and managing the experimental data. ESL has designed and supervised the biosensor project along with advising and reviewing the manuscript. All authors read and approved the final manuscript.

\section{Author details}

${ }^{1}$ Advanced Energy Systems and Microdevices Laboratory, Department of Mechanical and Industrial Engineering, New Jersey Institute of Technology, 200 Central Avenue, Rm MEC 327, Newark, NJ 07102-1982, USA. ${ }^{2}$ Provost Summer Research Intern at New Jersey Institute of Technology \& Tenafly High School, Tenafly, NJ, USA. ${ }^{3}$ Department of Electrical and Computer Engineering, New Jersey Institute of Technology, Newark, NJ 07102, USA.

\section{Acknowledgements}

The authors acknowledge the research support from New Jersey Institute of Technology (NIT). This research is carried out in part at the Center for Functional Nanomaterials, Brookhaven National Laboratory, which is supported by the U.S. Department of Energy, Office of Basic Energy Sciences, under Contract No. DE-SC0012704.

\section{Competing interests}

The authors declare that they have no competing interests.

\section{Availability of data materials}

The datasets used and/or analysed during the current study are available from the corresponding author on reasonable request.

\section{Funding}

This research is supported by the National Science Foundation Fund (Grant ID: NSF IIP-1643861).

\section{Publisher's Note}

Springer Nature remains neutral with regard to jurisdictional claims in published maps and institutional affiliations.

Received: 2 November 2018 Accepted: 7 January 2019

Published online: 17 January 2019

\section{References}

1. C. Berggren, B. Bjarnason, G. Johansson, Capacitive biosensors. Electroanal. Int. J. Devoted Fundam. Pract. Asp. Electroanal. 13(3), 173-180 (2001)

2. V. Tsouti, C. Boutopoulos, I. Zergioti, S. Chatzandroulis, Capacitive microsystems for biological sensing. Biosens. Bioelectron. 27(1), 1-11 (2011)

3. S. Kumar, S. Kumar, M.A. Ali, P. Anand, V.V. Agrawal, R. John, S. Maji, B.D. Malhotra, Microfluidic-integrated biosensors: prospects for point-of-care diagnostics. Biotechnol. J. 8(11), 1267-1279 (2013)

4. A. Bange, H.B. Halsall, W.R. Heineman, Microfluidic immunosensor systems. Biosens. Bioelectron. 20(12), 2488-2503 (2005)

5. D. Itoh, F. Sassa, T. Nishi, Y. Kani, M. Murata, H. Suzuki, Droplet-based microfluidic sensing system for rapid fish freshness determination. Sens. Actuators B Chem. 171, 619-626 (2012)

6. S.M. Hanash, S.J. Pitteri, V.M. Faca, Mining the plasma proteome for cancer biomarkers. Nature 452(7187), 571 (2008)

7. A.P. Turner, Biosensors-sense and sensitivity. Science 290(5495), 1315-1317 (2000)

8. C. Yu, J. Irudayaraj, Multiplex biosensor using gold nanorods. Anal. Chem. 79(2), 572-579 (2007)

9. R. Vaidyanathan, L.M. Van Leeuwen, S. Rauf, M.J. Shiddiky, M. Trau, A multiplexed device based on tunable nanoshearing for specific detection of multiple protein biomarkers in serum. Sci. Rep. 5, 9756 (2015) 
10. E. Zeidan, S. Li, Z. Zhou, J. Miller, M.G. Sandros, Single-multiplex detection of organ injury biomarkers using SPRi based nano-immunosensor. Sci. Rep. 6, 36348 (2016)

11. C. Huang, C. Tsou, The implementation of a thermal bubble actuated microfluidic chip with microvalve, micropump and micromixer. Sens. Actuators A 210, 147-156 (2014)

12. D. Erickson, X. Liu, R. Venditti, D. Li, U.J. Krull, Electrokinetically based approach for single-nucleotide polymorphism discrimination using a microfluidic device. Anal. Chem. 77(13), 4000-4007 (2005)

13. H.J. Lee, T.T. Goodrich, R.M. Corn, SPR imaging measurements of 1-D and 2-D DNA microarrays created from microfluidic channels on gold thin films. Anal. Chem. 73(22), 5525-5531 (2001)

14. E.K. Sackmann, A.L. Fulton, D.J. Beebe, The present and future role of microfluidics in biomedical research. Nature 507(7491), 181 (2014)

15. L. Malic, D. Brassard, T. Veres, M. Tabrizian, Integration and detection of biochemical assays in digital microfluidic LOC devices. Lab Chip 10(4), 418-431 (2010)

16. W. Wang, T.B. Jones, Moving droplets between closed and open microfluidic systems. Lab Chip 15(10), 2201-2212 (2015)

17. X. Ma, Y. Wu, S. Jin, Y. Tian, X. Zhang, Y. Zhao, L. Yu, X.J. Liang, Gold nanoparticles induce autophagosome accumulation through size-dependent nanoparticle uptake and lysosome impairment. ACS Nano 5(11), 8629-8639 (2011)

18. S. Loyprasert, P. Thavarungkul, P. Asawatreratanakul, B. Wongkittisuksa, C. Limsakul, P. Kanatharana, Label-free capacitive immunosensor for microcystin-LR using self-assembled thiourea monolayer incorporated with Ag nanoparticles on gold electrode. Biosens. Bioelectron. 24(1), 78-86 (2008)

19. R.A. Sperling, W.J. Parak, Surface modification, functionalization and bioconjugation of colloidal inorganic nanoparticles. Philos. Trans. R. Soc. Lond. A Math. Phys. Eng. Sci. 368(1915), 1333-1383 (2010)

20. Z. Altintas, S.S. Kallempudi, Y. Gurbuz, Gold nanoparticle modified capacitive sensor platform for multiple marker detection. Talanta 118, 270-276 (2014)

21. X. Li, M. Yu, Z. Chen, X. Lin, Q. Wu, A sensor for detection of carcinoembryonic antigen based on the polyaniline-Au nanoparticles and gap-based interdigitated electrode. Sen. Actuators B Chem. 239, 874-882 (2017)

22. J.M. Pingarrón, P. Yáñez-Sedeño, A. González-Cortés, Gold nanoparticlebased electrochemical biosensors. Electrochim. Acta 53(19), 5848-5866 (2008)

23. S. Sarojini, A. Tamir, H. Lim, S. Li, S. Zhang, A. Goy, A. Pecora, K.S. Suh, Early detection biomarkers for ovarian cancer. J. Oncol. 2012 (2012)

24. A.M. Whited, K.V. Singh, D. Evans, R. Solanki, An electronic sensor for detection of early-stage biomarker/s for ovarian cancer. BioNanoScience 2(4), 161-170 (2012)

25. J.S. Daniels, N. Pourmand, Label-free impedance biosensors: opportunities and challenges. Electroanalysis 19(12), 1239-1257 (2007)

26. J.M. Goddard, D. Erickson, Bioconjugation techniques for microfluidic biosensors. Anal. Bioanal. Chem. 394(2), 469 (2009)

27. R. Raghav, S. Srivastava, Core-shell gold-silver nanoparticles based impedimetric immunosensor for cancer antigen CA125. Sens. Actuators B Chem. 220, 557-564 (2015)

28. Z. Zou, J. Kai, M.J. Rust, J. Han, C.H. Ahn, Functionalized nano interdigitated electrodes arrays on polymer with integrated microfluidics for direct bio-affinity sensing using impedimetric measurement. Sens. Actuators A 136(2), 518-526 (2007)

29. H.E. Ayliffe, A.B. Frazier, R.D. Rabbitt, Electric impedance spectroscopy using microchannels with integrated metal electrodes. J. Microelectromech. Syst. 8(1), 50-57 (1999)

30. E.W. Washburn, The dynamics of capillary flow. Phys. Rev. 17(3), 273 (1921)

31. S.H. Tan, N.T. Nguyen, Y.C. Chua, T.G. Kang, Oxygen plasma treatment for reducing hydrophobicity of a sealed polydimethylsiloxane microchannel. Biomicrofluidics 4(3), 032204 (2010)

32. B.B. Nunna, D. Mandal, S. Zhuang, E.S. Lee, Innovative point-of-care (POC) micro biochip for early stage ovarian cancer diagnostics. Sens. Transducers 214(7), 12-20. http://www.sensorsportal.com/HTML/DIGEST/P_2934. htm (2017)
33. W. Limbut P. Kanatharana, B. Mattiasson. P. Asawatreratanakul P. Thavarungkul, A reusable capacitive immunosensor for carcinoembryonic antigen (CEA) detection using thiourea modified gold electrode. Anal. Chim. Acta 561(1-2), 55-61 (2006)

34. S.S. Kallempudi, Y. Gurbuz, A nanostructured-nickel based interdigitated capacitive transducer for biosensor applications. Sens. Actuators B Chem. 160(1), 891-898 (2011)

35. D. Mandal, B.B. Nunna, S. Zhuang, S. Rakshit, E.S. Lee, Carbon nanotubes based biosensor for detection of cancer antigens (CA-125) under shear flow condition. Nano Struct. Nano Objects 15, 180-185 (2018)

36. B.B. Nunna, D. Mandal, S. Zhuang, E.S. Lee, A standalone micro biochip to monitor the cancer progression by measuring cancer antigens as a pointof-care (POC) device for enhanced cancer management. in Healthcare Innovations and Point of Care Technologies (HI-POCT), (IEEE, Amsterdam, 2017), pp. 212-215

37. C. Berggren, G. Johansson, Capacitance measurements of antibody-antigen interactions in a flow system. Anal. Chem. 69(18), 3651-3657 (1997)

38. Y. Cai, G. Jiang, J. Liu, Q. Zhou, Multiwalled carbon nanotubes as a solid-phase extraction adsorbent for the determination of bisphenol A, 4-n-nonylphenol, and 4-tert-octylphenol. Anal. Chem. 75(10), 2517-2521 (2003)

39. H. A Abdulbari, E. A Basheer, Investigating the enhancement of microfluidics-based electrochemical biosensor response with different microchannel dimensions. Curr. Anal. Chem. 13(5), 361-369 (2017)

40. B.B. Nunna, S. Zhuang, E.S. Lee, Flow control mechanism of capillary driven flow in microchannel using non-mechanical forces. in APS Meeting Abstracts (2016)

41. B.B. Nunna, S. Zhuang, E.S. Lee, Influence on capillary flow of human blood in PDMS micro channels due to various surface treatments. in ASME ICNMM Abstracts (2016)

42. B.B. Nunna, E.S. Lee, Point-of-care (POC) micro biochip for cancer diagnostics. in Proceedings of the TechConnect World Innovation Conference and Expo, (2017), pp. 14-17

43. B.B. Nunna, S. Zhuang, I. Malave, E.S. Lee, Ovarian cancer diagnosis using micro biochip. in NIH-IEEE 2015 Strategic Conference on Healthcare Innovations and Point-of-Care Technologies for Precision Medicine, (PCHT15-0056), (2015), pp. 9-10

44. B.B. Nunna, S. Zhuang, E.S. Lee, Hemorheology in PDMS micro channel with varied surface roughness. in APS Meeting Abstracts, (2015)

45. E.S. Lee, B. B. Nunna, United States Patent Application 15/811186. Washington, DC: U.S. Patent and Trademark Office. Biomarker detection and self-separation of serum during capillary flow, (2018)

46. E.S. Lee, B.B. Nunna, United States Patent Application PCT/ US2017/038554. Washington, DC: U.S. Patent and Trademark Office. Microfluidic Diagnostic Assembly, (2017)

47. E.S. Lee, B.B. Nunna, United States Patent Application PCT/ US2018/018316. Washington, DC: U.S. Patent and Trademark Office. Enhanced Sensitivity and Specificity for Point-of-Care (POC) Micro Biochip, (2018)

48. G. Raschke, S. Kowarik, T. Franzl, C. Sönnichsen, T.A. Klar, J. Feldmann, A. Nichtl, K. Kürzinger, Biomolecular recognition based on single gold nanoparticle light scattering. Nano Lett. 3(7), 935-938 (2003)

\section{Submit your manuscript to a SpringerOpen ${ }^{\circ}$ journal and benefit from:}

- Convenient online submission

- Rigorous peer review

- Open access: articles freely available online

- High visibility within the field

- Retaining the copyright to your article

Submit your next manuscript at $\boldsymbol{\nabla}$ springeropen.com 